\title{
EL COMPORTAMIENTO \\ DEL GASTO PUBLICO EN ESPAÑA \\ DURANTE LA SEGUNDA REPUBLICA, $1931-1935^{*}$
}

\author{
NITEVISS GARCIA SANTOS \\ Universidad complutenso de Madrid \\ PABLC) MARTIN AC:FÑA \\ Iniversidad de Alcalí \\ 1. Vundación Empresa Pública
}

\section{INTRODUCCION}

EI estudio de la conomía y de la políica coonómica durante la Segun da República sigue despertando entre nosotros un gran interés. I:l advenimiento del nuevo régimen coincidió con el peor año de la depresión mundial iniciada en 1929 y durante su corla existencial tuve que sobrevivir en circunstancias económicas muy desfaverables. En sus cocritus, los políticos re. publicanos hicieron notiar este desafortunade sucesu, destacando cómo la crisis internacional limitaba seriamente su caparidad de acción'. Naruralmente, al adverso escenario económico de la decadal de 1920 hubieral cenido menor trascendencia si no hubiese sido por las exageradals expectativas de incjoras sociales y económicass que había suscitado la llegalda al poder de los partides de izquierda y de los grupos republicanos?

Diversos autores han señalado que, además de la crisis internacional. cuya correa de trasmisión fue el sector exterior, la economía española se vio afectada negativamente por una caída de la demanda interior, resultado de la adopción de políticas macroeconómicas erróneas. El hecho de que el prin. cipal objetivo de las auroridades económicas de la época fuera la estabilidad del tipo de cambio, ha llevado al atribuir las causas del estancamiento interno y de la recesión industrial, más que a factores externos, a una política monetaria restrictiva y a una política financiera ortodoxa, cuando las circunstancias

$:$ Agradecemos a F. Comin y (i. Tortella las obscrvaciones yue han hecho a distintos borradores de este trabajo. Pero la responsabilidad por crrores y omisiones es sólo nuestra.

' Véasc, por ejemplo, Indalecio Prieto (1967), vol. 1, pp. 164-165.

Parafraseando la conocida lista de Salvador Madariaga (1931), del nuevo régimen se esperaba la resolución de muchos problemas tradicionales del país: el problema de la propiedad de la tierra, el problema de las libertades civiles, el problema del Ejército, el problema de la educación, el problema de la Iglesia, el problema de las nacionalidacles y. por último, aunque no por ello menos importante, el problema de la modernización cconómica. Como puede comprenderse, todas estas exigencias recaían, en última instancia, sobre los recursos de la llacienda pública. Sobre las tareas a las que se enfrentaba la República, desde la perspectiva de otro contemporáneo, véase también Gil Robles (1968), pp. +1 't se'g 
aconscjaban todo lo contrario; esto es, con sus acciones los políticus republicanos lejos de compensar los efectos de la crisis, tendieron a agravarlos ${ }^{3}$.

En varios trabajos, Comín y Martín Aceña, conjuntamente y por separado, han puesto de relieve lo equivocado de tales argumentaciones. Ni la polítical monetaria fue restrictiva (deflacionista) como para provocar una contracción en la demanda interna, ni la política fiscal invirtió la tendencia de años anteriores ". Además, es erróneo asignar al listado un papel protagonista en la determinación de la coyuntura económica, hasta el punto de atirmar que el estancamiento de los años treinta seal responsabilidad de sus acciones. El Estado de entonces era de dimensiones reducidas y los instrumentos de politica monetaria y presupuestaria a su disposición, bastante rudimentarios. Como recientemente ha reiterado Comín en su extenso estudio de la Hacienda española, no puede atribuirse al Estado la responsabilidad de los ciclos económicos. ni los de alza ni los de baja; el sector privado condicionaba de forma más importante que el público la evolución de la cconomía española ". Así pues. aún admitiendo que las autoridades económicas republicanas hubiesen adoptado una política macroeconómica restrictiva, el reducido tamaño del sector público en el conjuno de la economía limió la incidencia que dicha política pudo tener en la demanda agregada.

Pero es que, además, es preciso reconocer que la evolución de la economía española fue durante estos años (1930-35) menos negativa que para cl conjunto de la cconomía mundial. Aunque ciertamente el nivel de actividad cayó, ni la renta nacional en términos reales, ni el nivel de precios, registraron un brusco descenso durante los años de administración republicana; como en su momento observaron los contemporáneos y más recientemente han confirmado los autores que se han ocupado del periodo, España capeó la tempestad conómical de los años treinta mejor que la mayor parte de los países europeos".

Fn este artículo se pretende analizar la incidencia de la política fiscal sobre la demanda agregada durance la Segunda República. En particular se iniden los efectos del gassto público a través del cálculo del multiplicador de la renta y para ello se formula un modelo econométrico de la economía española para el período $1900-1935^{\circ}$. Otros autores también han tratado

" Véanse Florensa (1981). (García D) lgado (1980), Harrison (1983). Hernández An dreu (1980, 1986) y Palatox (1980, 1983).

- Martín Acena (1984), Marín Acena y Comin (1984) y Comin (1988 y 1989).

Comín (1988).

- Sobre la economía de la Seganda República, los trabajos más recientes son los de Hernánde\% Andreu (1986). Tedde (1986) y Comin (1989), cap. 5, pp. 777.845.

Se toman estos treinta y cinco años por la necesidad de considerar un periodo más amplio yue los años estrictos de nuestro interés, puesto yue el comportamiento de las macromagnitudes reflejan, además de las acciones coyunturales de política fiscal, movimien. tos a largo plazo. 
de medir la incidencia de la política presupuestaria en diversos países; al estudio pionero de Brown (1956) para Estados Unidos han seguido los de Pryor (1979) para Checoslovaquia, y los de Middleton (1981) y Broadberry' (1984) para Gran Bretaña. Nosotros, con una metodología similar, intentamos estimar la incidencia de la política presupuestaria, tratando de ver si el sector público se comportó de forma contractiva, contribuyendo a agravar la crisis, o, por el contrario, de manera expansiva y, por tanto, con efectos compensatorios y estabilizadores.

En la sección siguiente describimos el comportamiento de los principales componentes del Producto Nacional Bruto (PNB) entre 1900 y 1935, a partir de las series de que disponemos. A continuación se formula el modelo econométrico, se estiman sus parámetros y se calcula el multiplicador de la renta. En la sección cuarta se examina la evolución seguida por el gasto público y por la inversión privada durante la Segunda República y se compara con su tendencia durante la Dictadura de Primo de Rivera, en un intento de diferenciar los comportamientos discrecionales dentro de la evolución a largo plazo de ambas variables. El trabajo se cierra con una breve sección en la que se exponen las principales conclusiones.

\section{LA RENTA NACIONAI. Y SUS COMPONENTES *}

El cuadro 1, que recoge la evolución del PNB y de cada uno de sus componentes entre 1900 y 1935 , muestra que el ritmo de actividad descendió a partir de 1931, registrándose tasas de variación negativas para 1931, 1933 y 1935. En este último año, la producción total se situaba todavía por debajo del nivel alcanzado en 1929-30. Resulta obvio, por tanto, que la economía española durante la Segunda República atravesó, si no una profunda crisis, al menos una fase de estancamiento. A continuación, empleando la información que ofrecen los cuadros 1 y 2 , examinamos el comportamiento a largo plazo de los factores determinantes de la demanda agregada.

El consumo privado muestra una tendencia creciente y relativamente estable. De 1900 a 1920 su tasa de crecimiento medio se situó en torno a un 1,6 por 100 y cercana al 1,2 por 100 entre 1920 y 1930; de esta última fecha a 1935 se registró una talsa negativa próxima al 2,0 por 100. Por otra parte, la participación del consumo en el PNB presenta cierta tendencia a disminuir: pasa del 0,85 para el quinquenio 1900-1904 a un 0,76

- El examen de la evolución de la renta nacional y sus componentes también ha sido realizado por Carreras (1985) y por Comín (1987). 
para 1925.1929 , lo que supone una reducción de casi diez décimas, aunque posteriormente entre 1930 y 1931 vuelve a incrementar su participación.

La inversión total (privada y pública) aumenta de forma continua entre 1900 y 1930, aunque en ilgunos años se registran tasas de variación negativas; de 1900 a 1920 su crecimiento medio se sitúa en torno al 1,1 por 100 y acelera su ritmo de forma notable en el decenio siguiente, alcanzando una tasa del 6,6 por 100. Por el contrario, de 1930 a 1935 la demanda de inversión cae a una media anual del $-7,4$ por 100 . En cuanto a su participación en el PNB, constatamos que se mantiene hasta 1919 y a partir de esta fecha sube hasta alcanzar un máximo en 1929; de 1930 a 1935 la importancia de la inversión total en el PNB se reduce. El comportamiento de la inversión privada es el que determina, esencialmente, la demanda agregada de inversión: de 1900 a 1920 crece al 0,9 por 100 y de 1920 a 1930 al 6,4 por 100 , mientras que entre 1930 y 1935 se registra una tasa negativa del $-10,6$ por 100 .

Por lo que se refiere a las dos series que componen el total del gasto público (consumo público más inversión pública), observamos que ambas presentan una tendencia creciente para la totalidad del periodo (1900-1935) y su participación en el PNB aumenta de forma notable. De 1900 a 1920 el consumo público crece a una tasa del 2,1 por 100 ; de 1920 a 1930 , la tasa se sitúa en el 4,0 por 100 , y se eleva al 4,9 por 100 para $1930-1935$. En cuanto a la inversión pública, vemos que crece en los tres períodos que estamos considerando, con la particularidad de que su ritmo de incremento se acelera al pasar de una etapa a otra $(3,7$ por 100 para $1900-1920,9,1$ para 1920 1930 y 11,2 para $1930-1935)$.

Por último, comprobamos que el sector exterior presenta saldos positivos de 1900 a 1919 y negativos, con excepciones, a partir de esta última fecha; así pues, cabe suponer que mientras en el primer período los déficit transmitieron impulsos expansivos sobre la demanda agregada, en el segundo ejercieran una acción opuesta, esto es, tuvieran efectos claramente deflacionistas. 


\section{CUADRO 1}

Producto Nacional Bruto y sus componentes (1900-1935)

$(\mathrm{PNB}: 1913=100)$

\begin{tabular}{|c|c|c|c|c|c|c|c|}
\hline & $\begin{array}{l}\text { Consumo } \\
\text { privado }\end{array}$ & $\begin{array}{l}\text { Consumos } \\
\text { públicos }\end{array}$ & $\begin{array}{c}\text { Inversion } \\
\text { total }\end{array}$ & $\begin{array}{l}\text { Saldo } \\
\text { cxterior : }\end{array}$ & $P N B=$ & $\begin{array}{l}\text { Inversión } \\
\text { publica }\end{array}$ & $\begin{array}{l}\text { Inversió, } \\
\text { privada }\end{array}$ \\
\hline$\ldots \ldots \ldots$ & 73,6 & 5,2 & 8,5 & 1.6 & 88.9 & 8,2 & 0,3 \\
\hline $\begin{array}{lllll}\ldots & \ldots & \ldots & \ldots\end{array}$ & 84,9 & 5,2 & 7.7 & 0.7 & 98,5 & 7.3 & 0,4 \\
\hline$\ldots \ldots$ & $\boldsymbol{M , 0}$ & 5,3 & 6,6 & I.t & 103,3 & 6,2 & 0.3 \\
\hline 1903 & 87.6 & 5,1 & 7,2 & 1,5 & 101,5 & 6,8 & 0.4 \\
\hline 1904 & 75,1 & 5,4 & 6,8 & 1,8 & 89,1 & 0,3 & 0,5 \\
\hline 19015 & 75,5 & 5,1 & 6,0 & 1,2 & 87,9 & 5,5 & 0,6 \\
\hline$\ldots \ldots \ldots$ & 89,7 & 5,6 & 6.7 & 1,1 & 103.1 & 6,2 & 0,5 \\
\hline $1907 \ldots \ldots \ldots$ & 73,2 & 5.6 & 7,3 & 2.4 & 88,5 & 6,9 & 0.4 \\
\hline $1908 \ldots \ldots \ldots$ & 85.7 & 5,7 & 8,2 & 1.8 & 101,3 & 7.6 & 0.6 \\
\hline $\begin{array}{lllll} & \ldots & \ldots & \ldots & \ldots\end{array}$ & 84.7 & 6.6 & 8.0 & 2,4 & 101,7 & 7,3 & 0.7 \\
\hline $\begin{array}{lllll} & \ldots & \ldots & \ldots & \ldots\end{array}$ & 86,6 & 6,7 & 8,0 & 2.6 & 103.9 & 7.3 & 0,8 \\
\hline$\ldots \ldots$ & 87.0 & 7.4 & 8.7 & 1.9 & 105.0 & 8.1 & 0.6 \\
\hline 1912 & 88.4 & 7.4 & 4,7 & 3.1 & 108,6 & 8.6 & 1.1 \\
\hline 1913 & 75.5 & $x_{, 11}$ & 11,4 & +.5 & 100,0 & 11.2 & 0,7 \\
\hline IyIt & $x+, x$ & 8,4 & 8,8 & 1,0 & 102,9 & 7.8 & 1.0 \\
\hline 1415 & $x+x$ & 10.8 & 7.3 & 5.5 & 1118.4 & 0.5 & 0.8 \\
\hline$\ldots \ldots \ldots$ & 93.1 & 7.7 & 7.2 & 6,4 & $11+, 9$ & 6,6 & 0.6 \\
\hline$\ldots \ldots \ldots \ldots$ & $\$ 3,2$ & 6.5 & 6.3 & 7.7 & $103, \overline{7}$ & 5.8 & 01.5 \\
\hline$\ldots \ldots \ldots$ & y.t & 5.4 & 6,9 & 5.7 & 111.3 & 6,4 & 0,5 \\
\hline$\ldots \ldots \ldots$ & 86.5 & 6.3 & 7.8 & 7,8 & 108,4 & 7.4 & 0.5 \\
\hline ...... $\ldots$ & 101,3 & 7,9 & 10.6 & -0.1 & 119,7 & 9,9 & 0,7 \\
\hline $\begin{array}{lllll}\ldots & \ldots & \ldots & \ldots\end{array}$ & 96,1 & 12,1 & 11,1 & -0.8 & 118,5 & 10,0 & 1,1 \\
\hline $\begin{array}{lllll}\ldots & \ldots & \ldots & \ldots \\
\end{array}$ & 88.0 & 12,9 & 10,5 & $-2,0$ & 109,3 & 9,0 & 1,5 \\
\hline $\begin{array}{lllll}\ldots & \ldots & \ldots & \ldots\end{array}$ & 97.7 & 11.6 & 13,4 & -1.3 & 121,4 & 12,0 & 1,4 \\
\hline $1924 \ldots \ldots \ldots \ldots$ & 86,4 & 11,9 & 14,1 & 0,2 & 113,1 & 12,6 & 1,6 \\
\hline $1925 \ldots \ldots \ldots \ldots$ & 101,6 & 11,9 & 16,1 & 0,6 & 130,2 & 14,7 & 1,4 \\
\hline $1926 \ldots \ldots \ldots \ldots$ & 98,0 & 12,6 & 16.2 & 1,2 & 127,9 & 14,8 & 1,3 \\
\hline $1927 \ldots \ldots \ldots \ldots$ & 97,8 & 12.3 & 18,0 & -0.8 & 127,4 & 15,5 & 2,5 \\
\hline $1928 \ldots \ldots \ldots \ldots$ & 107,0 & 12.3 & 19,9 & $-0,9$ & 138,4 & 16,2 & 3,7 \\
\hline $1929 \ldots \ldots \ldots \ldots$ & 99,5 & 12,1 & 22,9 & $-2,4$ & 132,1 & 18,9 & 4,0 \\
\hline $1930 \ldots \ldots \ldots \ldots$ & 114,2 & 11,8 & 20,4 & 0,4 & 146,9 & 18,6 & 1,8 \\
\hline $1931 \ldots \ldots \ldots$ & 89.8 & 11.5 & 15.5 & 0,0 & 116,9 & 13,4 & 2,1 \\
\hline $1932 \ldots \ldots \ldots \ldots$ & 123.5 & 12,3 & 13.3 & $-1,2$ & 147.9 & 10,6 & 2,7 \\
\hline $\begin{array}{lllll}\ldots & \ldots & \ldots & \ldots \\
\end{array}$ & 103,9 & 14,0 & 12,6 & -0.8 & 129,9 & 8,7 & 3.9 \\
\hline $193+\ldots \ldots \ldots \ldots$ & 115,9 & 14,5 & 13,4 & -1.6 & 142.2 & 9,4 & 4.1 \\
\hline $1935 \ldots \ldots \ldots \ldots$ & 103.7 & 15,1 & 14,2 & $-2,4$ & 130,1 & 11,0 & 3,2 \\
\hline
\end{tabular}

$\therefore$ Exportaciones menos importaciones.

$\because$ Producto Nacional Bruto.

Fuente: Carretas (1985). 


\section{CUADRO 2}

Participación media del consumo, de la inversión

$y$ del gasto público en el PNB

(En porcentajes)

\begin{tabular}{|c|c|c|c|}
\hline & $\begin{array}{c}\text { Consumo } \\
\text { privado }\end{array}$ & $\begin{array}{c}\text { Inversión } \\
\text { privada }\end{array}$ & $\begin{array}{c}\text { Gasto } \\
\text { público }\end{array}$ \\
\hline $\begin{array}{llllllll}1900-1904 & \ldots & \ldots & \ldots & \ldots & \ldots & \ldots & \ldots \\
1905-1909 & \ldots & \ldots & \ldots & \ldots & \ldots & & \ldots \\
1910-1914 & \ldots & \ldots & \ldots & \ldots & \ldots & \ldots & \ldots \\
1915-1919 & \ldots & \ldots & \ldots & \ldots & \ldots & \ldots & \ldots \\
1920-1924 & \ldots & \ldots & \ldots & \ldots & \ldots & \ldots & \ldots \\
1925-1929 & \ldots & \ldots & \ldots & \ldots & \ldots & \ldots & \ldots \\
1930-1935 & \ldots & \ldots & \ldots & \ldots & \ldots & \ldots & \ldots \\
193 & \ldots & \ldots & \ldots\end{array}$ & $\begin{array}{l}0,85 \\
0,85 \\
0,81 \\
0,80 \\
0,80 \\
0,76 \\
0,80\end{array}$ & $\begin{array}{l}0,043 \\
0,041 \\
0,049 \\
0,035 \\
0,055 \\
0,071 \\
0,048\end{array}$ & $\begin{array}{l}0,077 \\
0,081 \\
0,095 \\
0,155 \\
0,225 \\
0,213 \\
0,251\end{array}$ \\
\hline
\end{tabular}

Nota: El cuarto componente de la demanda agregada no incluido en este cuadro es el sector exterior.

Fuente: Carreras (1985).

\section{UN MODELO PARA LA ECONOMIA ESPAÑOLA, 1900-1935}

Acabamos de comprobar que el gasto público registró una evolución creciente durante todo el período que hemos considerado, incluidos los años de administración republicana; de hecho, puede comprobarse cómo a partir de 1931 aumenta en mayor medida que el resto de las magnitudes, lo que permite suponer que ejerciera notables efectos expansivos que compensaron la caída de otras variables, principalmente la inversión privada. En esta sección tratamos, precisamente, de contrastar este argumento y de medir el efecto expansivo del gasto público sobre la renta nacional.

Para ello estimamos, en primer lugar, un modelo explicativo del comportamiento de la economía española para el período 1900-1935. El modelo está formado por tres ecuaciones". La primera es una ecuación de consumo, en la que la tasa de crecimiento del consumo en términos reales depende de la tasa de crecimiento de la renta real en el mismo período $\left(\frac{\dot{\mathrm{Y}}}{\mathrm{P}}\right)_{b}$, del

- Todos los cálculos en esta sección y en las siguientes se han hecho con las magnitudes expresadas en términos reales. 
diferencial del tipo impositivo respecto al año anterior $\left(\Delta t_{t-1}\right)$ y del diferencial entre la tasa de crecimiento de la renta y del consumo del período anterior $\left(\dot{\mathrm{Y}}_{1-1}-\dot{\mathrm{C}}_{1-1}\right)^{10}$.

La segunda es una ecuación de importaciones, cuya tasa de crecimiento, en términos reales es función de la tasa de crecimiento de la renta real del período actual $\left(\frac{\dot{\mathrm{Y}}}{\mathrm{P}}\right)$, de la tasa de crecimiento de los precios en el período anterior $\left(\dot{P}_{t-1}\right)$, del diferencial de tipos de cambio en el período anterior $\left(\Delta t_{t-1}\right)$, de la tasa de importaciones, en términos reales, del período anterior $\left(\frac{\dot{M}}{P}\right)_{t-1}, y$, finalmente, de una variable ficticia (D) que recoge los efectos de la Primera Guerra Mundial sobre el comercio exterior".

La tercera ecuación es la identidad del PNB ( $Y=C+I+G+X-M)$, expresada en términos reales y en tasas de crecimiento:

$$
\begin{aligned}
\left(\frac{\dot{Y}}{P}\right)= & \frac{\dot{C}}{P}\left(\frac{C}{Y}\right)+\frac{\dot{I}}{P}\left(\frac{I}{Y}\right)+\frac{\dot{G}}{P}\left(\frac{G}{Y}\right)+ \\
& +\frac{\dot{X}}{P}\left(\frac{X}{Y}\right)-\frac{\dot{M}}{P}\left(\frac{M}{Y}\right)^{12}
\end{aligned}
$$

10 La ecuación de consumo se obtiene a partir del supuesto de una función en la que al consumo depende de la renta disponible ( $Y$ ); esto es, $C=f\left(Y_{d}\right)$, donde $Y_{d}=(Y-t Y)$; luego $C=f(1-t) Y$. Expresando dicha ecuación en tasas de crecimiento, tenemos que

$$
C_{\mathrm{t}}=\Delta \ln C, \text { y } \dot{Y}_{\mathrm{dt}}=\Delta \ln (1-\mathrm{t}) \mathrm{Y}_{\mathrm{t}}=-\Delta \mathrm{t}+\Delta \ln \mathrm{Y}_{\mathrm{t}} \simeq-\Delta \mathrm{t}+\dot{\mathrm{Y}}_{\mathrm{t}}
$$

luego $\dot{C}_{t}=g\left(\dot{Y}_{d t}\right)=g\left(-\Delta t+\dot{Y}_{t}\right)$. El último término de la ecuación de consumo es de corrección de error: el exceso de la tasa de crecimiento de la renta sobre la tasa de crecimiento del consumo en el período anterior influye en el consumo del período presente.

"No presenta ningún problema suponer yue las importaciones sean una función del nivel de renta; también parece lógico suponer que dependan del nivel de precios interiores y del tipo de cambio. Además de la variable artificial que se incluye, se experimentó con otra dummy que recogiese los efectos del arancel de 1922, pero su influencia nunca resultó significativa. Por último, al incluir como variable independiente la tasa de crecimiento de las importaciones del período anterior, se quiso recoger el carácter tendencial de dicha variable.

1: A esta exuación se llega a partir de la identidad contable del PNB, Y $=\mathrm{C}+\mathrm{I}+$ $+G+X-M$, expresándola en tasas de crecimiento y en términos reales; esto es:

$$
\left(\frac{\dot{Y}}{P}\right)=\left(\frac{Y_{t}-Y_{t-1}}{Y_{t}}\right) \frac{1}{\dot{P}}=\left(Y_{t}-Y_{t-1}\right) \frac{1}{\dot{P} Y_{t}}
$$


La estimación conjunta del modelo se ha hecho por método trietápico, que produce estimadores consistentes y asintóticamente eficientes, y los resultados se exponen en el cuadro 3. Los estadísticos muestran un alto grado de asociación y no existe correlación serial de primer orden en los residuos. La estimación del modelo es, por tanto, válida. En primer lugar, hemos realizado un test de estabilidad con objeto de comprobar si los períodos 1900 . 1930 y 1931-1935 pueden considerarse estructuralmente homogéneos o, por el contrario, entre ambos existe una ruptura que sugiera la conveniencia de analizarlos separadamente ${ }^{13}$. Según nuestras estimaciones, el valor obtenido para el test de estabilidad es de 10,55 , que comparado con el teórico de una $\%^{2}$ de 43,77 , sugiere que se puede aceptar la hipótesis de estabilidad para 1931. 1935; esto es, puede afirmarse que existe continuidad entre este período y el anterior (1900-1930), no registrándose, por tanto, ninguna ruptura de carácter estructural.

$y$, sustituyendo $Y_{t} \subset Y_{t-1}$ por sus componentes, obtenemos:

$\left(\frac{\dot{Y}}{P}\right)_{t}=\left[\left(C_{t}+I_{t}+G_{t}+X_{t}-M_{t}\right)-\left(C_{t-1}+I_{t-1}+G_{t-1}+X_{t-1}-M_{t-1}\right)\right]\left(\frac{1}{\dot{P} Y_{t}}\right)$

y agrupando términos:

$$
\begin{aligned}
\left(\frac{\dot{Y}}{P}\right)_{t}=\left[\left(C_{t}-C_{t-1}\right)\right. & +\left(I_{t}-I_{t-1}\right)+\left(G_{t}-G_{t-1}\right)+\left(X_{t}-X_{t-1}\right)- \\
& \left.-\left(M_{t}-M_{t-1}\right)\right] \frac{1}{\dot{P} Y_{t}}
\end{aligned}
$$

y de aquí obtenemos que

$$
\left(\frac{\dot{\mathrm{Y}}}{\mathrm{P}}\right)=\left(\frac{\dot{\mathrm{C}}}{\mathrm{P}}\right)_{1} \frac{\mathrm{C}_{\mathrm{i}}}{\mathrm{Y}_{1}}+\left(\frac{\dot{\mathrm{I}}}{\mathrm{P}}\right)_{1} \frac{\mathrm{I}_{\mathrm{i}}}{\mathrm{Y}_{1}}+\left(\frac{\dot{\mathrm{G}}}{\mathrm{P}}\right)_{1} \frac{\mathrm{G}_{\mathrm{t}}}{\mathrm{Y}_{1}}+\left(\frac{\dot{\mathrm{X}}}{\mathrm{P}}\right), \frac{\mathrm{X}_{1}}{\mathrm{Y}_{1}}-\left(\frac{\dot{\mathrm{M}}}{\mathrm{P}}\right)_{1} \frac{\mathrm{M}_{1}}{\mathrm{Y}_{1}}
$$

${ }^{13}$ En este caso, el tratamiento conjunto del período 1900.35 conduciria a no tener en cuenta rasgos diferenciales. Io que llevaría a conclusiones equivocadas. 


\section{CUADRO 3}

Modelo para la economia española, 1900.1935

(Estimación trietápica)

$$
\begin{aligned}
& \left(\frac{\dot{C}}{P}\right)=0,93\left(\frac{\dot{r}}{P_{1}}\right)-0,002 \Delta t_{1}+0,43(\dot{Y}-\dot{C})_{1-1} \\
& \text { (16,5) } \quad(-2,93) \quad(3,15) \\
& N=34 ; S S R=0,026 ; R^{\prime}-0,98 ; 1 D W=2,04 \\
& \left.(\stackrel{\dot{M}}{P})=0,97\left(\frac{\dot{Y}}{P}\right)+1,3 \dot{P}_{1}, \ldots 0,025\right),(3,15) P\left(1 c_{1-1}+0,53\left(\frac{\dot{M}}{P}\right)-0,23 \mathrm{D}\right. \\
& N-34: S . S R-0,17 ; R^{\prime}=0,67 ; D W=2,15
\end{aligned}
$$

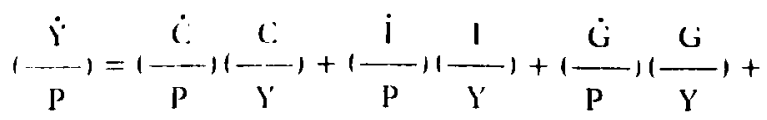

$$
\begin{aligned}
& \therefore\left(\frac{\dot{X}}{P}\right)\left(\frac{\mathrm{X}}{\mathrm{Y}}\right)-\left(\frac{\dot{\mathrm{M}}}{\mathrm{P}}\right)\left(\frac{\mathrm{M}}{\mathrm{Y}}\right)
\end{aligned}
$$

NOTAS

$$
\dot{Y} . \dot{C}, \dot{P},\left(\frac{\dot{I^{\prime}}}{P}\right),\left(\frac{\dot{C}}{P}\right),\left(\frac{\dot{M}}{P}\right),\left(\frac{\dot{I}}{P}\right),\left(\frac{\dot{G}}{P}\right),\left(\frac{\dot{X}}{P}\right)
$$

respectivamente, son la tasa de crecimiento del PNB nominal, la tasa de crecimiento del consumo privado nominal, la tasa de crecimiento del nivel de precios, y las tasas de crecimiento en términos reales del PNB, del consumo privado, de las importaciones, de la inversión privada, del gasto público y de las exportaciones. D es una variable artificial que toma el valor 1 enire 1914 y 1918, y 0 en el resto; Jic, a el diferencial del tipo de cambio pescta-libra del período con el período anterior; $J t_{t}$ es el diferencial del tipo impositivo directo del período con el del período anterior. La serie del PNB que se utiliza en el modelo es la estimada por Alcaide (1976); empleamos ésta, en lugar de la serie de Carreras, para evitar problemas de multicolinealidad.

Las cifras en paréntesis son los estadisticos "t»" de Student; $N=$ número de observaciones: $S S R=$ error estandar de la ecuación; $R^{2}=$ coeficiente de determinación: $\mathrm{DW}=$ Durwin-Watson. 
Respecto a las estimaciones obtenidas observamus que el consumo depende muy estrechamente del nivel de renta real disponible y que los impuestos personales ejercen una influencia de poca importancia; la elasticidad del consumo respecto a la renta es de 0,93 . La ecuación de importaciones muestra que las dos variables más significativas son el nivel de precios interiores y la renta nacional; la elasticidad con respecto a los precios es mayor que uno, lo que sugiere que sus variaciones producen una respuesta más que proporcional de la demanda de importaciones; en cuanto a la elasticidad con respecto a la renta, comprobamos que se aproxima a la unidad, por lo que sus variaciones provocaban cambios casi proporcionales en las importaciones. La influencia del tipo de cambio es reducida y la variable artificial nos indica que la Guerra Mundial provocó una caída de las exportaciones europeas hacia España.

Para conocer el efecto de un cambio unitario en la tasa de crecimiento del gasto público sobre la tasa de variación de la renta nacional, hemos de calcular el multiplicador de la renta. Resolviendo el modelo y teniendo en cuenta la fórmula del multiplicador en una economía abierta, llegamos a la expresión:

$$
\mathrm{m}=\frac{1}{1-0,93 \frac{\mathrm{C}}{\mathrm{Y}}+0,98 \frac{\mathrm{M}}{\mathrm{Y}}}
$$

Tanto para el cálculo del multiplicador de la inversión privada como para el cálculo del multiplicador gasto público tenemos en cuenta la respectiva participación de ambas magnitudes en la renta, de modo que el primero se obtiene a partir de la expresión $m \cdot 1 / Y$ y el segundo a partir de $m \cdot G / Y$.

Las estimaciones se ofrecen en el cuadro 4 y se presentan en promedios quinquenales. Observamos que el multiplicador del gasto público muestra cambios sustanciales en el período 1900-1935: la tendencia es creciente entre 1900 y 1920 , se reduce ligeramente de 1925 a 1930 y vuelve a aumentar entre 1931 y 1935. Así pues, estos primeros resultados sugieren que los efectos de la política fiscal fueron más intensos (expansivos) durante la Segunda República que en la década anterior. En cuanto al multiplicador de la inversión comprobamos que se mantiene relativamente constante, aunque alcanza sus valores máximos en el período 1925-1935. 


\section{CUADRO 4}

Multiplicador del gasto público y de la inversión privada

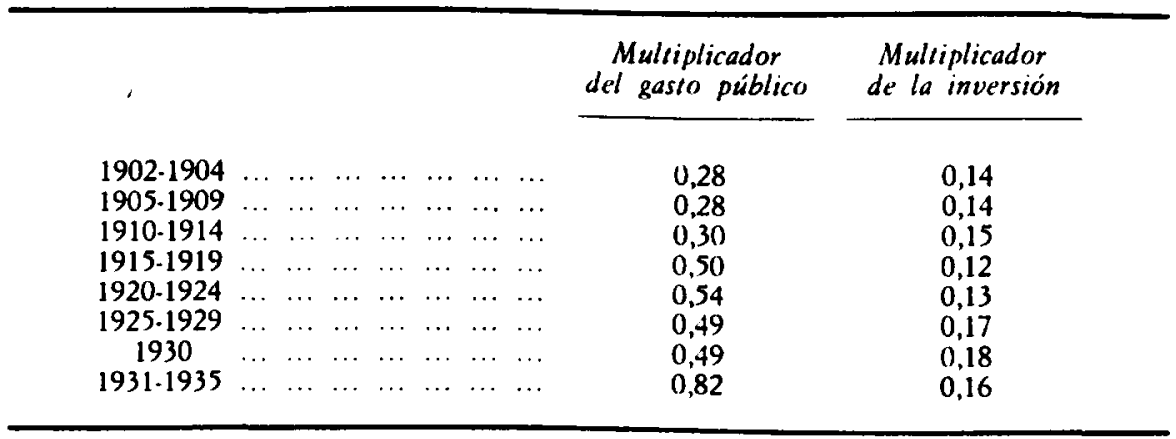

Nota: Recuérdese que el cálculo de los multiplicadores se hace a partir de las expre. siones $\mathrm{mG} / \mathrm{Y}$ y $\mathrm{m} \mathrm{I} / \mathrm{Y}$ (véase texto en la sección 3 ); esto es lo que explica que los valores estimados sean inferiores a la unidad.

Fuente: Cuadro 3 (Modelo).

\section{SIMULACIONES CON EL GASTO PUBLICO Y CON LA INVERSION PRIVADA}

Utilizando el modelo que hemos estimado para la economía española, podemos obtener una serie de tasas estimadas de crecimiento del PNB en términos constantes, y su mayor o menor divergencia con los valores originales indican la existencia o no de sucesos aleatorios e imprevistos no recogidos en las ecuaciones del modelo. En el cuadro 5 se comparan ambas series, las tasas originales y las estimadas a partir del modelo, y se comprueba que la evolución del PNB queda bien explicada por los argumentos de los que se ha hecho depender y que son los que figuran en el modelo. Los sucesos aleatorios no son significativos, ni siquiera durante los años iniciales de la recesión económica de los años treinta (1931 y 1932). 


\section{CUADRO 5}

Tasas de crecimiento del PNB original y estimado (En porcentajes)

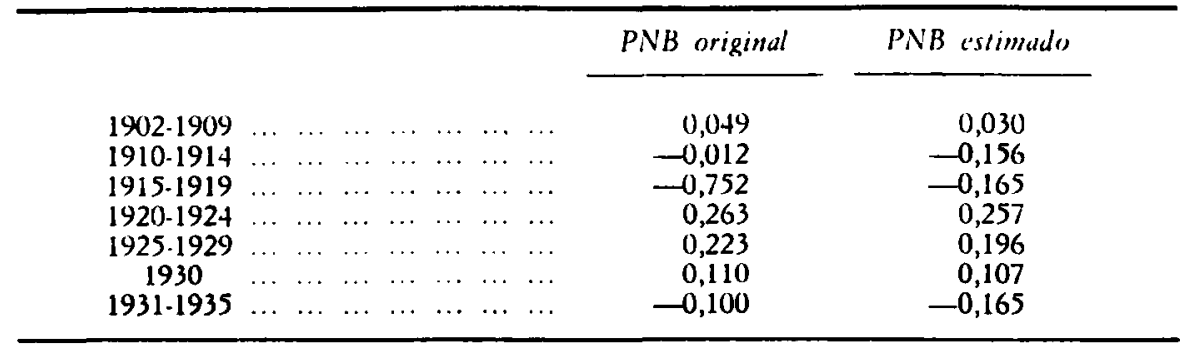

Fuente: Cuadro 3 (Modelo).

\section{i) Gasto público e inversión privada nulos}

Con el modelo estimado se pueden efectuar simulaciones suponiendo que, primero, el gasto público hubiese sido cero y, segundo, que lo hubiese sido la inversión privada. En ambos casos podemos obtener tasas de crecimiento del PNB en ausencia de cada una de estas dos variables, y la diferencia entre la tasa estimada y la simulada nos proporcionaría la contribución de la in. versión y del gasto público al crecimiento de la renta. Los cálculos, para distintos períodos, se ofrecen en el cuadro 6.

\section{CUADRO 6}

Tasas de crecimiento del PNB simuladas

(En porcentajes)

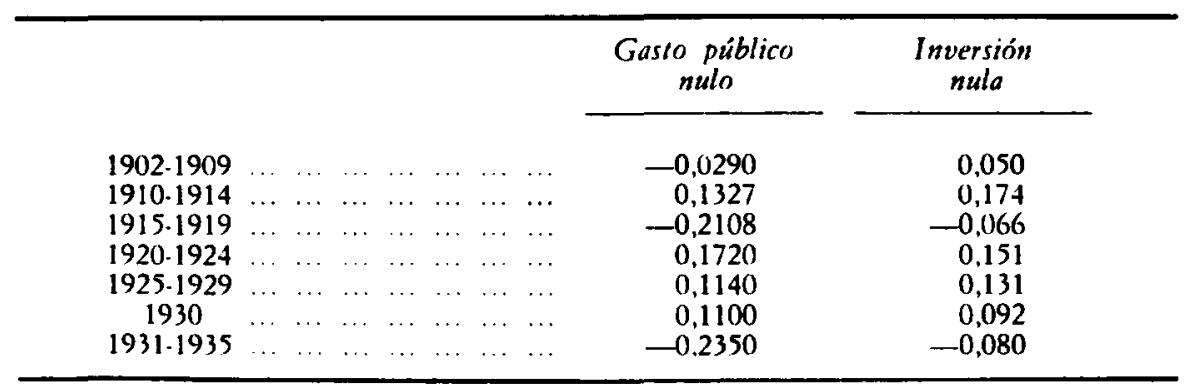

Fufute: Cuadro 3 (Modelo). 
En ausencia de gasto público, entre 1931 y 1935 la tasa de crecimiento del PNB real hubiese sido un $+2,2$ por 100 menor de la que efectivamente se registró, y para 1925-1929 y 1920-1924 hubiese sido un 41,8 y un 33,1 por 100 menor, respectivamente. En 1930 el gasto público fue, sin embargo, ligeramente contractivo, yal que sin su presencia el PNB real habria crecido a una tasa situada un 2,8 por 100 por encima de la efectiva. Estos resul. tados están en consonancia con los puestos de manifiesto por el multiplicador de la renta, que nos mostraba el efecto de un incremento del gasto público sobre la tasa de crecimiento del PNB. Los valores del multiplicador para cl periodo 1900-1935 reflejaban un efecto expansivo del gasto público sobre el PNB cada ve\% mayor, que se debian tanto al multiplicador en sí mismo (m) como al hecho de que aumentase la participación del gasto público en el PNB $(G / Y)$. Ahora, en la simulación que acabamos de realizar, se confirma el poder expansivo del mulcipliciador.

Sin inversión privada el PNB real simulado of rece un panorama distinto. Durante los años 1920-1929 la contribución aparece con signo positivo, lo que sugiere que su ausencia hubiera hecho caer la tasa de crecimiento del PNB real en torno at un +1$)$ por 100); de 19201-192+ lat inversión privada contribuyó más al crecimiento del PNB que el gasto público, aunque en la segun. da mitad de los años veinte las sillación se invierte. $A$ partir de 1931 lat presencia de tasas de crecimicono negativas de la inversión privada hace que su contribución al crecimieno sea muy contractiva. Puede afirmarse yue si entre 1931-1935 no hubieral existido inversión privada, la tasa de crecimiento del PNB real hubiese sido un 51,5 por 100 mayor de la que en realidad se registró.

Gasto público e inversión privada se comportaron de manera opuesta durante la Segunda República '4. La inversión privada experimentó un comportamiento contractivo, mientras que el gasto público, al ser más expansivo que durante los años veinte, permitió compensar el descenso de las tasas de crecimiento del PNB en un to por 100. Así pues, la acción del gasto público fue estabilizadora, si bien no pudo contrarrestar del todo los efectos contractivos de la inversión privada.

ii) (iasto priblico a inersion privada seguin lis temdentad de $190(1)-1930$ $y$ de 1918.1930

Acabamos de comprobar que durante los anos treinta no se interrumpe la tendencia de crecimiento del gasto público y que, además, éste cstimula

it A esta mismat conchlosión llegaren, en sus trabajos respectivos, Carreras (1985) y Comin $(1987)$ 
en mayor medida que en años precedentes al Producto Nacional Bruto. Ahora bien, es preciso tener en cuenta que el gasto público, en un determinado periodo, no es sólo resultado de la política fiscal discrecional que se adopte en dicho período, sino que su evolución tiene también un componente tendencial o secular. En consecuencia, interesa distinguir si a partir de 1931 existe algún cambio de tendencia en el gasto público con respecto a la que se observa durante la Dictadura o. por el contrario, su evolución en los años treinta es una simple continuación de su comportamiento de años anteriores. En el epígrafe siguiente se aborda este problema, y el ejercicio se realiza tanto para el gasto público como para la inversión privada.

\section{Evolución según la lendencia de 1900-1930.}

Suponemos, en primer lugar, que durante los años 1931.1935 el gasto público ha seguido la misma tendencia de los años 1900-1930. Para conocer dicha tendencia efectuamos una regresión mínimo cuadrática frente al tiem. po (cuadro 7) y extrapolamos la estimación para 1931-1935. De las ecuaciones se deduce que durante los años 1931.1935 el gasto público creció a un ritmo superior de lo esperado de acuerdo con la tendencia de años precedentes. Así, si con el gasto extrapolado calculamos las tasas de crecimiento que habría seguido el PNB durante los años 1931.1935, observamos, con. forme a lo que cabia esperar, que el PNB habria conocido un mayor estan. camiento (su tasa de crecimiento hubiera sido menor).

\section{CUADRO 7}

Gasto público e inversión privada, según la tendencia de 1900-1930

A. Estimaciones mínimo cuadráticas:

$$
\begin{aligned}
& \left(\frac{\dot{\mathrm{G}}}{\mathrm{P}}\right)_{\mathrm{t}}=\underset{(3,16)}{35,11}+\underset{(3,48)}{2,4 t}+0,31\left(\frac{\dot{\mathrm{G}}}{(1,75) \mathrm{P}}\right)_{\mathrm{t}-1} \\
& N=30 ; \bar{R}^{:}=0,79 ; D W=2,19 ; F_{2,27}=54,78 ; Q_{2}=5,5 \\
& \left(\frac{1}{P}\right)=\underset{(1,96)}{0,38 t}+0,91\left(\frac{\mathrm{I}}{(16,2)}\right)_{t-1} \\
& N=30 ; \bar{R}^{:}=0,72 ; D W=1,79 ; F_{1,28}=75,04 ; Q_{2}=1,42
\end{aligned}
$$




\section{CUADRO 7 (Continuación)}

Gasto público e inversión privada, según la tendencia de 1900-1930

B. Tasas del crecimiento del PNB:

\begin{tabular}{|c|c|c|c|}
\hline \multirow[b]{2}{*}{ Periodo } & \multirow[t]{2}{*}{$\begin{array}{c}\text { Estimación según } \\
\text { el multiplicador } \\
\text { del modelo cuadro } 3\end{array}$} & \multicolumn{2}{|c|}{$\begin{array}{l}\text { Estimación extrapolando } \\
\text { la tendencia de 1900-1930 } \\
\text { (modelo cuadro } 7)\end{array}$} \\
\hline & & $\begin{array}{c}\text { 'n el gasto } \\
\text { publico }\end{array}$ & $\begin{array}{c}\text { in la } \\
\text { inversion }\end{array}$ \\
\hline 1931.1935 & $-0,165$ & $-0,279$ & $-0,040$ \\
\hline
\end{tabular}

Notas: "t» es la variable de tendencia temporal, y las demás variables y estadisticas responden a la nomenclatura definida en el cuadro 3 .

Realizando el mismo ejercicio para la inversión privada, esto es, suponiendo que su evolución hubiera seguido la tendencia de los años 1900-1930. lo que se deduce en este caso es que la inversión habría sido mayor que lo que efectivamente fue y el PNB habría experimentado una caída menor.

\section{Evolución según la tendencia de 1918-1930.}

Ahora suponemos que ambas variables, gasto público e inversión privada, hubieran seguido la tendencia correspondiente al período 1918-1930. La razón de elegir estos años es que constituyen un período de crecimiento homogéneo tras la guerra europea, recogiendo de forma más precisa lo acontecido durante la Dictadura de Primo de Rivera. En el cuadro 8 se recogen las ecuaciones que reflejan la tendencia del gasto público y de la inversión en dicho periodo. De acuerdo con las estimaciones, si durante los años 1931-1935 el gasto público real hubiera seguido la tendencia reflejada en los años 1918-1930, habría tenido un crecimiento medio similar al que en efecto tuvo y en con. secuencia el PNB habría crecido, empleando el gasto público predicho según dicha tendencia, a una tasa similar a la realmente registrada. 


\section{CUADRO 8}

Gasto público e inversión privada según la tendencia de 1918.1930

A. Estimaciones mínimo cuadraticas:

$$
\begin{aligned}
& \left(\frac{\dot{G}}{P}\right)_{1}=\underset{(3,5)}{3,8 t}+\underset{(1,4)}{0,29}\left(\frac{\dot{G}}{P}\right)_{t-1} \\
& N=12 ; \bar{R}^{2}=0,67 ; D W=1,9 ; F_{1,11}=23,7 ; Q_{2}=0,098 \\
& \left(\frac{\dot{I}}{p}\right)_{1}=\frac{-89,2+6,15 t}{(-13,5)(23,5)} \\
& N=13 ; \bar{R}^{:}=0,98 ; D W=2,18 ; F_{1,11}=554,0 ; Q_{2}=62
\end{aligned}
$$

\begin{tabular}{|c|c|c|c|}
\hline \multirow[b]{2}{*}{ Pariodo } & \multirow[t]{2}{*}{$\begin{array}{c}\text { Estimación según } \\
\text { el multiplicador } \\
\text { del modelo cuadro } 3\end{array}$} & \multicolumn{2}{|c|}{$\begin{array}{c}\text { Estimación extrapolando } \\
\text { la tendencia de 1918.1930 } \\
\text { (modelo cuadro 8) }\end{array}$} \\
\hline & & $\begin{array}{c}\text { en el gasto } \\
\text { puiblico }\end{array}$ & $\begin{array}{c}\text { en la } \\
\text { inversión }\end{array}$ \\
\hline 1931.1935 & $-0,165$ & $-0,168$ & 0,0091 \\
\hline
\end{tabular}

B. Tasas de crecimiento del PNB:

Los resultados que se deducen para la inversión privada son diferentes. Sabemos que en 1931, 1932 y 1933 se registraron tasas de variación negativas, a diferencia de los incrementos de años anteriores, por lo que si la inversión privada hubiese mantenido su tendencia del período 1918.1930, el PNB para 1931-1935 habría experimentado una tasa media de crecimiento positiva, muy al contrario de lo que acaeció.

En conclusión, el gasto público efectivo durante los años de la República fue superior al esperado, según la tendencia correspondiente a 1900-1930 y similar al que corresponde a la tendencia de 1918-1930. Su efecto sobre el crecimiento del PNB habría de considerarse expansivo en el primer caso y neutro en el segundo; esto es, en el primer caso las autoridades económicas habrían elevado discrecionalmente el gasto por encima de lo esperado, y en el segundo habrian seguido la misma política fiscal de la Dictadura. Por el contrario, la inversión privada durante los años 1931-1935 cayó respecto a 
los valores esperados, tanto si consideramos la de 1900).1930 como si lo hacemos con la de 1918-1930. Así pues, el hecho de que el gasto público fuera mayor que el esperado hizo que la recesión fuera menor; contrariamente, el hecho de que la inversión privada fuera menor provocó que la recesión ad. quiriera mayores dimensiones. La inversión privada fue, en definitiva, la causa del estancamiento económico de esos años.

Los resultados se confirman calculando los multiplicadores de la renta que resultarian según esas magnitudes predichas (cuadro 9). En efecto, si el gasto público hubiera seguido la tendencia marcada por el periodo 1900-1930. su efecto multiplicativo sobre la renta habría side menor que el que en realidad fue. Si hubiera seguido la tendencia de 1918-1930 habría sido prácticamente igual. Por el contrario, si la inversión privada hubiera seguido la tendencia de los años 1900-1930, su impacto sobre la renta habría sido mucho mavor y la diferencia habría aumentado si hubiera seguido la pauta de los años veinte. Así pues, el comportamiento de la inversión privada durante los años treinta fue más contractivo de lo esperado, tanto si se compara con su evolución del período $1900-1930$ como si se hace con respecto a la de los años 1920-1930.

\section{(UUADR 9}

Multiplicador de la remia con al gasto peiblico $v$ con la imversión privada extrapolados

$A$

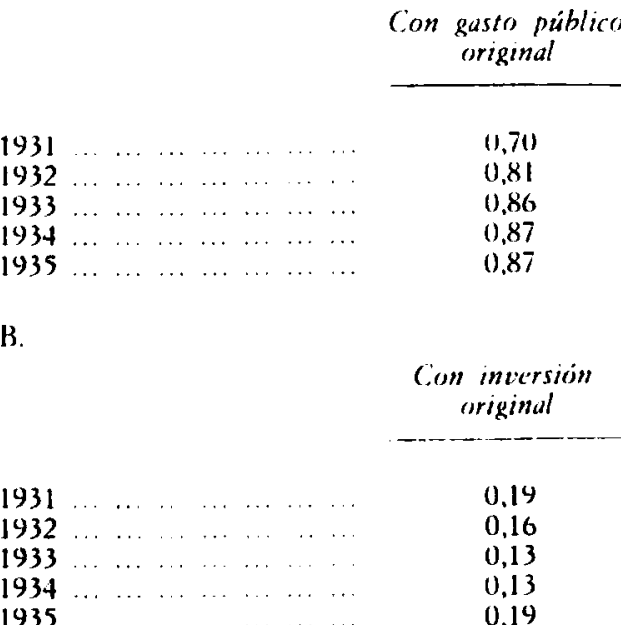

Con gasto público según tendencia $1900 \cdot 1930$

Con gasto público según tendencia 1918.1930

\begin{tabular}{|c|c|}
\hline 0,70 & 0,77 \\
\hline 01,68 & $(0,81$ \\
\hline 0,72 & 0,81 \\
\hline 0,72 & 0,85 \\
\hline 0.75 & $(0,87$ \\
\hline $\begin{array}{l}\text { Con inversion } \\
\text { segun tendencia } \\
1900.1930\end{array}$ & $\begin{array}{c}\text { Con inversion } \\
\text { segü tendencia } \\
1918.1930\end{array}$ \\
\hline 0,26 & 0,28 \\
\hline 0,28 & 0,30 \\
\hline 0,29 & 0,32 \\
\hline 0,29 & 0,34 \\
\hline 0,31 & 0,35 \\
\hline
\end{tabular}




\section{CONCLUSION}

El análisis cuantitativo que hemos llevado a cabo en las secciones anteriores establece, de forma definitiva, que la política fiscal española durante la Segunda República no tuvo carácter restrictivo, ni fue causa de la crisis industrial, ni tendió a agravar la situación económica. Por el contrario, la evolución del gasto público compensó, en parte, la caída de la inversión privada y de la demanda exterior. El nivel de gasto público no descendió y su tasa de crecimiento fue incluso superior a la esperada según su tendencia en años anteriores. La desaceleración en el ritmo de incremento del PNB debe atribuirse al comportamiento negativo de la inversión privada y del sector exterior, que, conjuntamente, ejercieron fuertes impulsos depresivos. La política fiscal contribuyó a que la crisis económica española fuese menor de lo que en otro caso hubiese sido.

Naturalmente, este resultado no fue producto de una política keynesiana avant la lettre. Como ha recordado Comín recientemente, la ortodoxia financiera no fue abandonada y los ejecutores de la política económica sólo estuvieron preocupados por un deseo: liquidar el presupuesto sin déficit. Pero, como también se señala por este mismo autor, la realidad de la política fiscal muchas veces difería de los deseos de los ministros de Hacienda ${ }^{15}$; esto es, los presupuestos se liquidaban con saldos negativos a pesar de los encargados del Tesoro. En definitiva, parece demostrado que cualesquiera que hubiesen sido los deseos o las intenciones de las autoridades fiscales republicanas, su acción quedó lejos de la ortodoxia financiera. La política presupuestaria de la Segunda República fue de carácter anticíclico.

\footnotetext{
"Comín (1988), pp. 847-848.
} 


\section{BIBLIOGRAFIA}

AlCaIDE, J. (1976): «Una revisión urgente de la seric de renta nacional española en el siglo XX", en Datos básicos para la historia financiera de España, 1850.1975. Madrid. Instituto de Estudios Fiscales.

Broadberry, S. N. (1984): "Fiscal Policy in Britain during the 1930s", Ficonomic History Review, núm. 37.

Brown, E. C. (1956): "Fiscal Policy in the Thirties: A Reappraisal». Americun liconomin Review, XLVI, núm. I.

Carreras, A. (1985): "(iasto Nacional Bruto y formación de capital en España, 1849.1958: Primer ensayo de estimación», en P. Martín Aceña y L. Prados de la Escosura. La Nucva Historia Económica e’n lispaña. Madrid, Teconos.

Comin, F. (1985): Fuentes cuantitativas para el estudio del serter publese en t:spañ. 1801-1980, Madrid. Instituto de Estudios Fiscales.

- (1987): "La economía española en el periodo de entreguerras", en J. Nadal, $\Lambda$. Ca. rreras y C. Sudrià, La economia española en el siglo XX: Ina peripectiva bistórica. Barcelona, Ariel.

- (1989): Hacienda y coomemia in la España comlemporánet. 1800 1936. Madrid. Ins tituto de Estudios Fiscales.

Estadisticas Históricas de España. Siglos XIX y XX, 1989 (coordinación de A. Carreras), Madrid, Fundación Banco Exterior

Fiorensa, S. 11981 !: «Los más relevantes aspectos económico-sociales al principio y al fin de la II República". en R. Calle, La Hacienda Publica e'n la II Republica. Madrid. Instituto de Estudios Fiscales.

García Delgado, J. L. (1980): «La política económica española de 1930: el error Argüelles», Papeles de Economia Española, núm. 1.

Gil. Robles, J. M. (1978): No fuc posible la paz, Barcelona, Editorial Planceta.

Harrison, J. (1983): "The Inter-war Depression and the Spanish Economy», Journal of European Economic History, núm. 2. Fall.

HeRnández ANDREU, J. (1980): Depresión económica en España, 1925-1934, Madrid Instituto de Estudios Fiscales.

- (1986): España y la crisis de 1929. Madrid, Espasa-Calpc.

Madariaca, S. (1931): España. Ensuyo de bistoria contemporanéa, Madrid.

Martín ACeña, P. (1984): La politica monetaria en España, 1919.1935, Madrid, Instituto de Estudios Fiscales.

Martín Aceña, P., y Comin, F. (1984): "La política monetaria y fiscal durante la Dictadura y la Segunda República», Papeles de Economia Española, núm. 20.

Martínez Méndez, P. (1983): «Nuevos datos sobre la evolución de la peseta entre 1900 y 1936 , en G. Anes, L. $\Lambda$. Rojo y P. Tedde, Historia económica y pensamiento social. Madrid, Alianza.

Midpleton, R. (1981): «The Constant Employment Budget Balance and British Budgetary Policy, 1919.39", Economic Hislory Review, vol. XXXIV, núm. 2.

Palafox, J. (1980): “La gran depresión de los años treinta y la crisis industrial espa. ñolaw, Investigaciones Económicus, núm. 11.

- (1983): "The Economy of Interwar Lurope: Fiscal Policy in Spain 1929.1936m, ponencia presentada al VIII Congreso Internacional de Historia Liconómica. Budapest.

Prifto, I. (1967): Convulsione's en España, México, Ediciones Oasis.

PRYOR, Z. P. (1979): "Czechoslovak Fiscal Policies in the Great Depression», Economic Historay Review, vol. XXXII, núm. 3.

Tedde de LorCA, P. (1986): «La economía en la Segunda República», en O. Ruiz Manjón, Historia de España. La Segunda República, Madrid, Rial.

Tortella, G., y Palafox, J. (1983): «Banca e industria en España, 1918-1936», Inves. tigaciones Económicas, núm. 20. 